\title{
Digital image correlation for structural health monitoring - a review
}

\author{
K Tharun Kumar Reddy ${ }^{*}$, Srikanth Koniki ${ }^{1}$ \\ *PG student, Civil Engineering Department, GRIET, Hyderabad, India, Email: tharunreddy79@gmail.com \\ ${ }^{1}$ Associate Professor, Civil Engineering Department, GRIET, Hyderabad, India, Email: sri.sri001@gmail.com
}

\begin{abstract}
This paper presents a detailed review of the use of digital image correlation on structural health monitoring. The durability of structures like bridges and buildings etc., need to monitor continuously. Structural health monitoring plays a vital role in assets and planning. Detailed knowledge on digital image correlation concerning structural health monitoring and civil engineering applications is presented in this paper.
\end{abstract}

\section{Introduction}

The process of implementing the damage detection techniques in engineering structures before full damage is termed structural health monitoring. The identification and analysing of deterioration in structure concerning age are done under structural health monitoring. Damage may occur in the materials results in a variation of the geometry of the structure [1]. Structural health monitoring shows the durability effect of the structure by analysing the periodical dynamic response measurements. The adverse effects on the structure depend on many natural environment conditions like an earthquake, temperature difference, ages, humidity effects etc., and some other depends on the properties of materials. In the civil engineering aspect structural health monitoring is necessary because the concrete is the material that will be affected by parameters like stress and strain, shrinkage, creep, concrete curing temperature [2]. Crack and damage detection is difficult in structural health monitoring. Every structure should be monitored for the benefit of society without affecting the environmental conditions.

The durability of the structure depends on strength crack detection. Structural members are affected by the strain and deformation in many engineering structures. The strain measurements to be more accurate because misleading strain values causes a major loss of financial and human lives [1]. To overcome these problems accurate methods are to be adopted. There are traditional methods available which measure strains, as a scratch strain gauge is used to find the strain in any particular location [3]. Electrical strain gauges are used for accurate results, but fixing the procedure of the gauge to the specimen is difficult [4]. Strain gauges will not provide accurate measurements in small strain analysis and are not suitable for surface deformations subjected to high temperature [5] and finite element analysis used rapidly [4]. Not all the traditional methods results are up to the mark in finding the strain and deformation measurements [5]. DIC is used to overcome the issues by the traditional methods and densiometric methods, [7]. For the high degree of accuracy measurement values, digital image correlation is to be used. DIC gives accurate full-field deformation measurement results. These will capture the very small strain values and finds the surface deformation at higher temperatures. It is a very cost-effective technique in structural health monitoring, which measures the full-field displacement and strain. DIC is used for any size specimen and can be tested for any material type specimens. DIC gives the analysed results of stress concentrators and strain. DIC can measure both very small and very large types of deformation strains. It is also applicable in real-time structure in various fields with more accuracy. Formatting the title, authors and affiliations

\section{Digital image correlation}

Peters and Ranson et al. [6] used DIC for the first time on experiment stress analysis. DIC is the one widely used technique for detecting damage in structural health monitoring. DIC is preferred in structural health monitoring because of its non-contact nature. The study of DIC gives the concentration of strain, deflection, and crack behavior in the structure. It is an optical technique that results in the measurement of full-field deformations which occurred at the surface of the specimen [17]. DIC is used in many research processes in various fields [31].

\footnotetext{
* Corresponding author: tharunreddy79@gmail.com
} 
This paper presents a detailed review of the use of digital image correlation on structural health monitoring. The schematic setup of DIC process is shown in figure 1 .

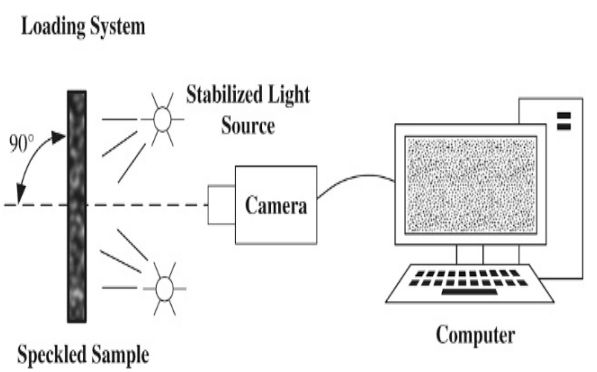

Fig. 1. Schematic Diagram

\section{Operating principle}

DIC works based on a mutual image correlational process. The correlation will be done for the deformed images concerning the one undeformed (reference) image. DIC can be done in both two- dimensional and three-dimensional analysis [9]. The speckle pattern dots are kept on the surface of the specimen which should cover the surface by more than fifty per cent of surface area DIC will divide the speckle pattern into the small grid subsets to analyse clearly; grids are square or rectangle in shape as shown in fig 2. Based on the displacements in the location of the variable speckle pattern points in the grid subset of an element gives the analysis of full-field displacement [24]. The correlation analysis of the images is done in the software by performing mathematical algorithms [28]. Algorithms are used in the correlation process to reduce the changes in the intensities. Zero-order shape functions are used for the subsets that undergo rigid body translation. Firstorder shape functions are used to represent the subsets that undergo translation, rotation, and normal and shear. Algorithms in DIC are used in analysing the different properties of shape functions. For sub-pixel accuracy in measurements, employment of grey values between pixel locations has been done before the image matching process. High order interpolation takes a long time for the image correlation process and gives more accuracy than low order interpolation [23]. Some interpolation methods, which are used, are bilinear interpolation [24], bi-cubic spline interpolation [25], and bi-cubic B spline interpolation [26]. In 1983 Sutton et al. [24] improved DIC by implementing bilinear interpolation. In $1985 \mathrm{chu}$ et al. [27] improved by introducing four parameters in
DIC like uniform translation, rigid body rotation, uniform finite strain test, constant angular velocity motion. Sutton et al. [28] modified DIC by introducing Newton- Raphson method. Hovis et al. [29] developed DIC by introducing a centroidal tracking algorithm. Vendroux and knauss et al. [30] optimized NewtonRaphson method and introduced Scanning Tunnelling Microscopy (STM). Jiang et al. [31] employed the integral image technique.

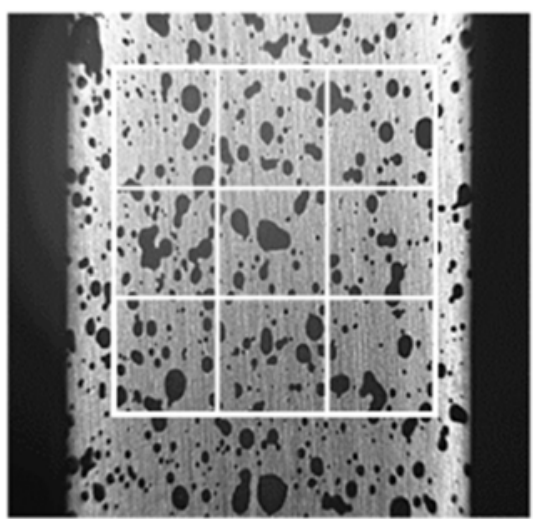

Fig 2. The concentrated speckle Grid pattern

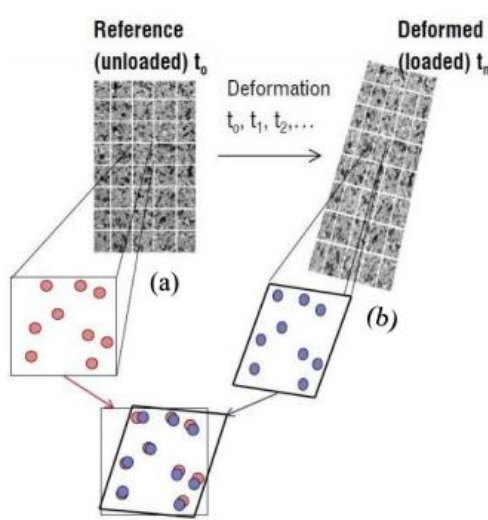

Fig. 3. Co-relation process of images

\section{Fundamental concepts of two- dimensional DIC}

The speckle pattern dots are kept on the surface of the specimen which should cover the surface by more than fifty per cent of surface area. A high-resolution camera is used for clear images. Deformed and undeformed specimen pictures are taken and compared by correlation process. DIC is a measurement technique that compares the changed parameters of the deformed captured images 
with the actual reference image. It will correlate many patterns of images such as dots figure 3 . The change in location of the dots will be measured and find in the pixel values. These measured pixel values give the strain and deformation measurement values. The pixel values are converted into standard mathematical values. And many other parameters can be found out by the DIC method with graphical representation [45]. The correlation process is done in the software by performing mathematical algorithms programs. DIC helps in;

1. Identifying the deterioration on the specimen,

2. locates the damages,

3. identities the type of deterioration and,

4. Intensity and severity of the damage.

\section{Applications of DIC in Structural Health Monitoring.}

DIC was first applied in the mechanic's field for analyzing two-dimensional measurements [6]. In recent years DIC technique was used in analyzing the deformation behavior of many materials used in different fields like mechanical, civil, biotechnical fields [9]. DIC method is used for analyzing displacements of a specimen under loading conditions. DIC techniques are commonly applicable in fracture mechanics [10]. In a study of plasticity deformation of the notch of the specimen which was subjected to tensile loading [11]. DIC technique is extending day by day and applicable to the crack propagation study to measure stress intensity factors [12]. In 2002, the DIC technique is applied in the fatigue analysis [13]. DIC is applicable in identifying the mechanical parameters of the specimens [14]. DIC is applicable for testing the materials which can use in paving roads [32]. DIC is applicable for cement paste for finding settlement and shrinkage cracks [15]. DIC is applicable in analyzing the interfacial bonding properties [16]. DIC is capable of measuring the in-plane surface deformation [17].

\section{Errors Affecting DIC measurements}

\subsection{Accuracy and precision}

Finding the difference between systematic error and random error is important. In the DIC technique accuracy and precision are to be taken and considered. The modification of software parameters results in larger changes. Conversion of pixels to be handled [18]

\section{2 patterns}

DIC analysis depends on pattern surface. The speckle pattern is virtually translated into correlate zero strain condition. To reduce the errors effecting the DIC is based on relation between the facet size and mean speckle size. Small site arrangement in more area results in occurrence of less areas and vice versa [19].

\subsection{Captured images}

Errors affects the images which are captured by the camera such as excess sound, thermal sound and electromagnetic sounds of relative measurement chain images from out plane displacements during loading [20].

\subsection{Correlation process}

During the correlation process the position of the variable function of point gives the strain and displacement parameters. While correlation process the mathematical analyzing algorithms program will be done. This to be minimized by performing the process with more accuracy [21].

\section{Literature survey}

This section presents a comprehensive review of the research studies conducted on DIC. The review also focuses on recent studies on the application of DIC for structural health monitoring. Jian- weizoh et al. [22] examined the application of DIC in measuring the mechanical properties with high accuracy of packaging lightweight materials which are too soft by using DSMS (Digital Speckle Measurement System) and results in the detailed mechanical properties determined with high accuracy. Wenzhitang et al. [36] studied the formation of continuous and discontinuous cracks on the concrete structures by using subset restore and adaption method of DIC and results in the handling of both small and large translation, rotational cracks with high accuracy. Congsun et al. [35] studied the influence of DIC in measuring the full field three-dimensional deformation measurements using the scheimpflug camera and Lagrangian strain magnitude maps and results in the displacement magnitude, change in surface area for different loadings. Ming-hisang shih et al. [37] researched on the structural monitoring of old buildings under shock and vibration conditions using Approximate story damage index and interstory drift mode shape in DIC by shearing the steel structures and results in accurate monitoring. Evin Dildar Dzaye et al. [15] examined the settlement and shrinkage cracks of fresh cement mortar by using digital image correlation and compared the results with classical LVDT point transducers and it results in the DIC have accurate results than traditional methods. Zhe Li et al. [33] researched in monitoring the rusting procedure of reinforced bars in concrete and acceleration of chloride ions by using electromagnetic sensors and DIC and tests are checked by core drilling method in concrete and observed the relationship between the frequency of the corrosion and loading. Bora Gencturk et al. [43] compared the results of strains and deflections of prestressed concrete I beam with conventional displacement transducers and digital image correlation. It stated that DIC results are very 
accurate and provide detailed information than other conventional methods. Mohammed shbeh et al. [44] examined the research on substitute material of bone with different morphologies of titanium foam in biomedical applications by using DIC and results in showing the low yield stress in cubic space holder than spherical space holder. W.A.Scrivens et al.[38] explored the methods in patterning the metal films at microscale and nanoscale by measuring in a scanning microscope. It results in nanoscale remodeling in metal films with random patterns with different morphologies. H.Jin et al. [39] studied micro-scale deformation by using scanning electron microscopy and DIC. It was applied for strain concentration of crack during ductile fracture test and it resulted in measuring small deformations. S.Rizo patron et al.[40] examined the modal analysis of a helicopter rotor blade using DIC. It results in finding the modal parameters with the accuracy of $0.2 \%$ to $10 \%$. Y.H.Wang et al.[41] applied the DIC technique for measuring the tensile strain in whole field sheet metal. The results obtained the true strain measurements by DIC which are accurate than average strain which was obtained by the traditional extensometer technique. T.L.Jin et al.[42] examined the study on thermal buckling behavior of circular aluminum plate using DIC and finite element analysis. It results in measuring the buckling temp from the temperature displacement curve. Obtained temperature values by DIC are accurate than experimental results.

\section{Conclusion}

The application of DIC has been reviewed in detail. Considering the damages in buildings, bridges etc by natural disasters is extremely worst condition. Testing and measuring the intensities in structures at an early stage is need for SHM. Different applications of digital image correlation have been studied and concluded in it. Advancement in digital image correlation is becoming huge in many fields. To increase the measurement accuracy and computational efficiency, DIC is preferred mostly. DIC in the civil engineering field for structural health monitoring is reviewed.

\section{References}

1. Wen, Sihai, and D. D. L. Chung. Cem and Con Res 29.3 (1999).

2. Chung, D. D. L. Jou of mat sci 36.6 (2001).

3. Haglage, Theodore L., and Howard A. Wood. Scratch strain gage evaluation. AIR FLI DYN LAB WRI -PAT AFB OH, 1969.

4. Khan, Akhtar S., and Xinwei Wang. Strain measurements and stress analysis. 2001.

5. A. Moteurs. "COM AND SUN GRO (GROWTH) PRO" Exper Mech 6 (2005): 8th. 